\title{
THE SPECTRAL GEOMETRY OF EINSTEIN MANIFOLDS WITH BOUNDARY
}

\author{
JEONGHYEONG PARK
}

\begin{abstract}
Let $(M, g)$ be a compact Einstein manifold with smooth boundary. Let $\Delta_{p, \mathcal{B}}$ be the realization of the $p$ form valued Laplacian with a suitable boundary condition $\mathcal{B}$. Let $\operatorname{Spec}\left(\Delta_{p, \mathcal{B}}\right)$ be the spectrum where each eigenvalue is repeated according to multiplicity. We show that certain geometric properties of the boundary may be spectrally characterized in terms of this data where we fix the Einstein constant.
\end{abstract}

\section{INTRODUCTION}

Let $\Delta_{p}$ be the Laplace-Beltrami operator acting on the space of smooth $p$ forms over a compact $m$ dimensional Riemannian manifold $M$ with smooth boundary. If the boundary is non-empty, then we impose boundary conditions defined by a suitably chosen operator $\mathcal{B}$ to define the realization $\Delta_{p, \mathcal{B}}$. Let $\nabla$ be the Levi-Civita connection of $M$ and let $e_{m}$ be the inward unit normal vector field on the boundary. Then, for example, Dirichlet and Neumann boundary conditions are defined by the corresponding Dirichlet and Neumann boundary operators:

$$
\mathcal{B}_{D} \phi:=\left.\phi\right|_{\partial M} \quad \text { and } \quad \mathcal{B}_{N} \phi:=\left.\nabla_{e_{m}} \phi\right|_{\partial M} \quad \text { for } \quad \phi \in C^{\infty}\left(\Lambda^{p} M\right) .
$$

In addition to the boundary conditions defined by these operators, there are also boundary conditions arising from index theory. Near the boundary, we decompose a differential form

$$
\phi=\sum_{I} \phi_{I} d y^{I}+\sum_{J} \psi_{J} d x^{m} \wedge d y^{J}
$$

into tangential and normal components. Absolute boundary conditions are then defined by the operator

$$
\mathcal{B}_{a} \phi:=\left.\left.\left\{\sum_{I} \partial_{m}^{x} \phi_{I} d y^{I}\right\}\right|_{\partial M} \oplus\left\{\sum_{J} \psi_{J} d y^{J}\right\}\right|_{\partial M} .
$$

Dually, we may use the Hodge $\star$ operator to define the relative boundary operator by setting:

$$
\mathcal{B}_{r} \phi:=\mathcal{B}_{a} \star \phi .
$$

In previous work [4, we extended a result of Patodi [3] from the context of closed Riemannian manifolds to the context of compact Riemannian manifolds with boundary. As we were interested in determining if the Einstein condition was spectrally determined, we worked in the context of manifolds of constant scalar curvature to show:

2000 Mathematics Subject Classification. 58J50.

Key words and phrases. totally umbillic boundary, totally geodesic boundary, minimal boundary, absolute boundary conditions, relative boundary conditions, Dirichlet Laplacian, Neumann Laplacian.

This work was supported by Korea Science and Engineering Foundation Grant (R05-2003-00010884-0). 
Theorem 1.1. Let $\left(M_{i}, g_{i}\right)$ be compact Riemannian manifolds with smooth boundaries and constant scalar curvatures $\tau_{i}$ for $i=1,2$. Let $\mathcal{B}$ define either Dirichlet or Neumann boundary conditions. Assume $\operatorname{Spec}\left(\Delta_{p, \mathcal{B}}\right)\left(M_{1}\right)=\operatorname{Spec}\left(\Delta_{p, \mathcal{B}}\right)\left(M_{2}\right)$ for $p=0,1,2$. Then if $\left(M_{1}, g_{1}\right)$ is Einstein, then $\left(M_{2}, g_{2}\right)$ is Einstein and $\tau_{1}=\tau_{2}$.

In this paper, instead of studying the geometry of the interior, we turn our attention to the geometry of the boundary. Motivated by Theorem 1.1 we shall assume henceforth that the manifolds under consideration are Einstein and we shall fix the Einstein constant, or, equivalently, the scalar curvature $\tau$.

We recall some basic definitions. Let indices $i, j$ range from 1 to $m$ and index a local orthonormal frame $\left\{e_{i}\right\}$ for the tangent bundle of $M$. Near the boundary, we further normalize the frame and assume that $e_{m}$ is the inward unit geodesic normal vector field. Let indices $a, b$ range from 1 to $m-1$ and index the induced local orthonormal frame $\left\{e_{a}\right\}$ for the tangent bundle of the boundary.

We adopt the Einstein convention and sum over repeated indices. Let $L$ be the second fundamental form and let $R_{i j k l}$ be the Riemann curvature tensor. The normalized mean curvature $\kappa$, the Ricci tensor $\rho$, and the scalar curvature $\tau$ are then given by:

$$
\kappa:=L_{a a}, \quad \rho_{i j}:=R_{i k k j}, \quad \text { and } \quad \tau:=\rho_{i i} .
$$

Since $M$ is Einstein, $\rho=\lambda g$ where $\lambda$ is the Einstein constant. This implies that $\tau=$ $m \lambda$. Thus fixing the Einstein constant is equivalent to fixing the scalar curvature.

Definition 1.2. We say that the boundary of $(M, g)$ is:

(1) totally geodesic if the second fundamental form vanishes identically. Equivalently, this means that if a geodesic in $M$ is tangent to the boundary at a single point, then the geodesic stays in $\partial M$.

(2) minimal if the normalized mean curvature vanishes identically. Equivalently, this means that the volume of the boundary is infinitesimally stationary.

(3) totally umbillic if at each point of the boundary, the second fundamental form has only one eigenvalue; the eigenvalue in question is allowed to vary with the point of the boundary.

(4) strongly totally umbillic if the the eigenvalue in (3) is independent of the boundary point chosen.

We can now state the main results of this paper. We first consider both Dirichlet and Neumann boundary conditions:

Theorem 1.3. For $i=1,2$, let $\left(M_{i}, g_{i}\right)$ be compact Einstein manifolds with smooth boundaries. Assume that $\tau_{1}=\tau_{2}$ and that

$$
\begin{aligned}
& \operatorname{Spec}\left(\Delta_{0, \mathcal{B}_{D}}\right)\left(M_{1}\right)=\operatorname{Spec}\left(\Delta_{0, \mathcal{B}_{D}}\right)\left(M_{2}\right), \quad \text { and } \\
& \operatorname{Spec}\left(\Delta_{0, \mathcal{B}_{N}}\right)\left(M_{1}\right)=\operatorname{Spec}\left(\Delta_{0, \mathcal{B}_{N}}\right)\left(M_{2}\right)
\end{aligned}
$$

where $\mathcal{B}_{D}$ and $\mathcal{B}_{N}$ define Dirichlet and Neumann boundary conditions, respectively. Then:

(1) If $\partial M_{1}$ is totally geodesic, then $\partial M_{2}$ is totally geodesic.

(2) If $\partial M_{1}$ is minimal, then $\partial M_{2}$ is minimal.

(3) If $\partial M_{1}$ is totally umbillic, then $\partial M_{2}$ is totally umbillic.

(4) If $\partial M_{1}$ is strongly totally umbillic, then $\partial M_{2}$ is strongly totally umbillic. 
In the previous Theorem, we studied two different boundary conditions for the operator $\Delta_{0}$. In the next Theorem, we study two different operators, $\Delta_{0}$ and $\Delta_{1}$, and impose either relative or absolute boundary conditions.

Theorem 1.4. For $i=1,2$, let $\left(M_{i}, g_{i}\right)$ be compact Einstein manifolds with smooth boundaries. Assume that $\tau_{1}=\tau_{2}$ and that

$$
\begin{aligned}
& \operatorname{Spec}\left(\Delta_{0, \mathcal{B}}\right)\left(M_{1}\right)=\operatorname{Spec}\left(\Delta_{0, \mathcal{B}}\right)\left(M_{2}\right), \quad \text { and } \\
& \operatorname{Spec}\left(\Delta_{1, \mathcal{B}}\right)\left(M_{1}\right)=\operatorname{Spec}\left(\Delta_{0, \mathcal{B}}\right)\left(M_{2}\right)
\end{aligned}
$$

where $\mathcal{B}$ denotes either relative or absolute boundary conditions. Then:

(1) If $\partial M_{1}$ is totally geodesic, then $\partial M_{2}$ is totally geodesic.

(2) If $\partial M_{1}$ is minimal, then $\partial M_{2}$ is minimal.

(3) If $\partial M_{1}$ is totally umbillic, then $\partial M_{2}$ is totally umbillic.

(4) If $\partial M_{1}$ is strongly totally umbillic, then $\partial M_{2}$ is strongly totally umbillic.

Here is a brief outline to the remainder of this paper. In Section 2 we review some facts concerning boundary geometry which we shall need. In Section 3] we recall some previous results concerning the heat trace asymptotics. In Section 4 . we use these results to complete the proof of Theorems 1.3 and 1.4

\section{THE GEOMETRY OF THE BOUNDARY}

Central to our proof of Theorems 1.3 and 1.4 is the following integral characterization of certain geometric properties. Let $d y$ denote the Riemannian measure on the boundary and let $d x$ denote the Riemannian measure on the interior. To simplify the notation, let

$$
f[M]=\int_{M} f(x) d x \quad \text { and } \quad f[\partial M]=\int_{\partial M} f(y) d y
$$

where $f$ is a scalar function.

Theorem 2.1. Let $M$ be a compact $m$ dimensional Riemannian manifold with smooth boundary $\partial M$.

(1) $\partial M$ is totally geodesic if and only if $L_{a b} L_{a b}[\partial M]=0$.

(2) $\partial M$ is minimal if and only if $L_{a a} L_{b b}[\partial M]=0$.

(3) $\partial M$ is totally umbillic if and only if $\left\{(m-1) L_{a b} L_{a b}-L_{a a} L_{b b}\right\}[\partial M]=0$.

(4) $\partial M$ is strongly totally umbillic if and only if there exists a constant $\mu$ so that $\left\{L_{a b} L_{a b}-2 \mu L_{a a}+\mu^{2}(m-1)\right\}[\partial M]=0$.

Proof. The first two assertions are immediate. To prove Assertion (3), we let $\left\{\kappa_{1}(y), \ldots, \kappa_{m-1}(y)\right\}$ be the eigenvalues of the second fundamental form at a point $y$ of the boundary. Then the second fundamental form is umbillic at $y$ if and only if $\kappa_{1}(y)=\ldots=\kappa_{m-1}(y)$ or equivalently if

$$
0=\sum_{i<j}\left(\kappa_{i}-\kappa_{j}\right)^{2} .
$$

Assertion (3) now follows since we have that

$$
\begin{aligned}
& \sum_{i} \kappa_{i}(y)^{2}=L_{a b} L_{a b}(y), \\
& \sum_{i, j} \kappa_{i}(y) \kappa_{j}(y)=L_{a a} L_{b b}(y), \quad \text { and } \\
& 0 \leq \sum_{i<j}\left(\kappa_{i}(y)-\kappa_{j}(y)\right)^{2}=(m-1) L_{a b}(y) L_{a b}(y)-L_{a a} L_{b b}(y) .
\end{aligned}
$$


Finally, to prove assertion (4), we note that the second fundamental form is $\mu$ times the identity at a point $y$ of the boundary if and only if

$$
0=\mid L-\mu \text { id }\left.\right|^{2}=L_{a b}(y) L_{a b}(y)-2 \mu L_{a a}(y)+(m-1) \mu^{2} .
$$

Since $|L-\mu \mathrm{id}|^{2}$ is non-negative, Assertion (4) now holds.

\section{Heat tRaCe ASymptotics}

To deal with Dirichlet, Neumann, and absolute boundary conditions in a common framework, it is useful to introduce the more general notion of mixed boundary conditions. Let $\chi$ be a self-adjoint endomorphism of $\left.\Lambda^{p}(M)\right|_{\partial M}$ so that $\chi^{2}=$ id. Let $\Pi_{ \pm}$be orthonormal projection on the \pm 1 eigenspaces of $\chi$. Let $S$ be an auxiliary endomorphism of range $\Pi_{+}$. The mixed boundary operator $\mathcal{B}_{\chi, S}$ is then defined by

$$
\mathcal{B}_{\chi, S} \phi:=\left.\left.\left\{\Pi_{+}\left(\phi_{; m}+S \phi\right)\right\}\right|_{\partial M} \oplus\left\{\Pi_{-} \phi\right\}\right|_{\partial M} .
$$

Example 3.1. Let $\mathcal{B}=\mathcal{B}_{\chi, S}$.

(1) If we take $\chi=-\mathrm{id}$, then $\mathcal{B}$ defines Dirichlet boundary conditions.

(2) If we take $\chi=\mathrm{id}$, then $\mathcal{B}$ defines Neumann boundary conditions.

(3) Let $\operatorname{ext}\left(e_{i}\right)$ denote left exterior multiplication by the covector $e_{i}$ and let $\operatorname{int}\left(e_{i}\right)$ be the dual operation, left interior multiplication by the covector $e_{i}$. Let $\Pi_{+}$be projection on $\Lambda(\partial M)$, let $\Pi_{-}$be projection on $\Lambda(\partial M)^{\perp}$, and let

$$
S=-\Pi_{+} \operatorname{ext}\left(e_{a}\right) \operatorname{int}\left(e_{b}\right) L_{a b} \Pi_{+} .
$$

Then $\mathcal{B}_{\chi, S}$ defines absolute boundary conditions, see, for example, the discussion in [2]. We note for future reference that

$$
\chi_{; a}=2 L_{a b}\left\{\operatorname{ext}\left(e_{b}\right) \operatorname{int}\left(e_{m}\right)+\operatorname{ext}\left(e_{m}\right) \operatorname{int}\left(e_{b}\right)\right\} .
$$

Let $\mathcal{B}=\mathcal{B}_{\chi, S}$ and let $e^{-t \Delta_{p, \mathcal{B}}}$ be the fundamental solution of the heat equation. The pseudo-differential calculus established by Seeley [5, 6] shows operator is of trace class and as $t \downarrow 0$ there is a complete asymptotic expansion with locally computable coefficients in the form:

$$
\operatorname{Tr}_{L^{2}} e^{-t \Delta_{p, \mathcal{B}}} \sim \sum_{n \geq 0} t^{(n-m) / 2} a_{n}\left(\Delta_{p}, \mathcal{B}\right) .
$$

Let ';' denote multiple covariant differentiation. The Weitzenböch formula permits us to express

$$
\Delta_{p} \omega=-\left(\omega_{; k k}+E_{p} \omega\right)
$$

where $E_{p}$ is a suitably chosen expression in the curvature tensor. For example, we have that

$$
E_{0}=0 \quad \text { and } \quad E_{1}\left(e_{i}\right)=-\rho_{i j} e_{j} .
$$

The following result is a special case of a more general result established by Branson and Gilkey [1].

Theorem 3.2. Let $M$ be a compact Riemannian manifold which has a smooth boundary $\partial M$. Let $\mathcal{B}=\mathcal{B}_{\chi, S}$ define mixed boundary conditions on $\Lambda^{p}(M)$.

(1) $a_{0}\left(\Delta_{p}, \mathcal{B}\right)=(4 \pi)^{-m / 2} \operatorname{Tr}\{\operatorname{id}\}[M]$.

(2) $a_{1}\left(\Delta_{p}, \mathcal{B}\right)=(4 \pi)^{-(m-1) / 2} \frac{1}{4} \operatorname{Tr}\{\chi\}[\partial M]$.

(3) $a_{2}\left(\Delta_{p}, \mathcal{B}\right)=(4 \pi)^{-m / 2} \frac{1}{6}\left\{\operatorname{Tr}\left\{6 E_{p}+\tau\right\}[M]+\operatorname{Tr}\left\{2 L_{a a}+12 S\right\}[\partial M]\right\}$.

(4) $a_{3}\left(\Delta_{p}, \mathcal{B}\right)=(4 \pi)^{-(m-1) / 2} \frac{1}{384} \operatorname{Tr}\left\{96 \chi E_{p}+16 \chi \tau-8 \chi \rho_{m m}\right.$ 


$$
\begin{aligned}
& +\left[13 \Pi_{+}-7 \Pi_{-}\right] L_{a a} L_{b b}+\left[2 \Pi_{+}+10 \Pi_{-}\right] L_{a b} L_{a b}+96 S L_{a a} \\
& \left.+192 S^{2}-12 \chi_{; a} \chi_{; a}\right\}[\partial M] .
\end{aligned}
$$

\section{Proof of Theorems 1.3 and 1.4}

Let $\mathcal{B}$ denote Dirichlet, Neumann, or absolute boundary conditions. The heat trace asymptotics $a_{n}\left(\Delta_{p}, \mathcal{B}\right)$ are spectral invariants. Consequently by Theorem 3.2

$$
\{\operatorname{vol}(M), \operatorname{vol}(\partial M)\}
$$

are spectral invariants. We have fixed the Einstein constant and set the scalar curvature $\tau=c$. Thus

$$
\tau[M], \quad \tau[\partial M], \quad \text { and } \quad \rho_{m m}[\partial M]
$$

are spectral invariants as well. The formula for $a_{2}$ then shows that

$$
L_{a a}[\partial M]
$$

is spectrally determined. In light of Theorem 2.1 to complete the proof of Theorems 1.3 and 1.4 it suffices to show

$$
L_{a a} L_{b b}[\partial M] \text { and } L_{a b} L_{a b}[\partial M]
$$

are spectrally determined by $\left\{\Delta_{0, \mathcal{B}_{D}}, \Delta_{0, \mathcal{B}_{N}}\right\}$, by $\left\{\Delta_{0, \mathcal{B}_{a}}, \Delta_{1, \mathcal{B}_{a}}\right\}$, or by $\left\{\Delta_{0, \mathcal{B}_{r}}, \Delta_{1, \mathcal{B}_{r}}\right\}$.

We shall supress the coefficients of certain invariants in what follows since they define invariants which are already known to be spectrally determined; we denote such coefficients by a generic symbol $\star$. We use the discussion in Example 3.1 the formulae in Equation [3.1] and Theorem 3.2 to compute:

$$
\begin{aligned}
a_{3}\left(\Delta_{0}, \mathcal{B}_{D}\right)=(4 \pi)^{(1-m) / 2} \frac{1}{384}\{ & \star \tau+\star \rho_{m m}-7 L_{a a} L_{b b} \\
& \left.+10 L_{a b} L_{a b}\right\}[\partial M], \\
a_{3}\left(\Delta_{0}, \mathcal{B}_{N}\right)=(4 \pi)^{(1-m) / 2} \frac{1}{384}\{ & \star \tau+\star \rho_{m m}+13 L_{a a} L_{b b} \\
+ & \left.2 L_{a b} L_{a b}\right\}[\partial M] .
\end{aligned}
$$

Since the coefficient matrix

$$
\left(\begin{array}{rr}
-7 & 10 \\
13 & 2
\end{array}\right)
$$

is non-singular, the invariants given in Equation (4.1) are spectral invariants as desired; Theorem 1.3 now follows.

To establish Theorem 1.4 we must perform similar computations for absolute and for relative boundary conditions.

Absolute boundary conditions are pure Neumann boundary conditions on 0 forms. By Example 3.1

$$
\begin{aligned}
& \operatorname{Tr}_{\Lambda^{1} M}\left\{\left[13 \Pi_{+}-7 \Pi_{-}\right] L_{a a} L_{b b}\right\}=(13 m-20) L_{a a} L_{b b}, \\
& \operatorname{Tr}_{\Lambda^{1} M}\left\{\left[2 \Pi_{+}+10 \Pi_{-}\right] L_{a b} L_{a b}\right\}=(2 m+8) L_{a b} L_{a b}, \\
& \operatorname{Tr}_{\Lambda^{1} M}\left\{96 S L_{a a}\right\}=\quad-96 L_{a a} L_{b b}, \\
& \operatorname{Tr}_{\Lambda^{1} M}\left\{192 S^{2}\right\}=\quad 192 L_{a b} L_{a b}, \\
& \operatorname{Tr}_{\Lambda^{1} M}\left\{-12 \chi_{: a} \chi_{: a}\right\}=\quad-96 L_{a b} L_{a b} \text {. }
\end{aligned}
$$


It is now an easy matter to use Theorem 3.2 to see that

$$
\begin{aligned}
a_{3}\left(\Delta_{0}, \mathcal{B}_{a}\right)=(4 \pi)^{(1-m) / 2} \frac{1}{384}\{ & \star \tau+\star \rho_{m m}+13 L_{a a} L_{b b} \\
& \left.+2 L_{a b} L_{a b}\right\}[\partial M] \\
a_{3}\left(\Delta_{1}, \mathcal{B}_{a}\right)=(4 \pi)^{(1-m) / 2} \frac{1}{384}\{ & \star \tau+\star \rho_{m m}+(13 m-116) L_{a a} L_{b b} \\
& \left.+(2 m+104) L_{a b} L_{a b}\right\}[\partial M]
\end{aligned}
$$

The desired result for absolute boundary conditions now follows as the determinant of the coefficient matrix

$$
\left(\begin{array}{rr}
13 & 2 \\
13 m-116 & 2 m+104
\end{array}\right)
$$

is 1584 which is different from zero.

Relative boundary conditions are Dirichlet boundary conditions on 0 forms. We use duality to see relative boundary conditions on 1 forms have the same spectral asymptotics as absolute boundary conditions on $m-1$ forms. On $m-1$ forms, we compute:

$$
\begin{aligned}
& \operatorname{Tr}_{\Lambda^{m-1} M}\left\{\left[13 \Pi_{+}-7 \Pi_{-}\right] L_{a a} L_{b b}\right\}=(-7 m+20) L_{a a} L_{b b}, \\
& \operatorname{Tr}_{\Lambda^{m-1} M}\left\{\left[2 \Pi_{+}+10 \Pi_{-}\right] L_{a b} L_{a b}\right\}=(10 m-8) L_{a b} L_{a b}, \\
& \operatorname{Tr}_{\Lambda^{m-1} M}\left\{96 S L_{a a}\right\}=\quad-96 L_{a a} L_{b b}, \\
& \operatorname{Tr}_{\Lambda^{m-1} M}\left\{192 S^{2}\right\}=\quad 192 L_{a a} L_{b b}, \\
& \operatorname{Tr}_{\Lambda^{m-1} M}\left\{-12 \chi_{: a} \chi_{: a}\right\}=\quad-96 L_{a b} L_{a b} .
\end{aligned}
$$

It is now an easy matter to use Theorem 3.2 to see that

$$
\begin{aligned}
a_{3}\left(\Delta_{0}, \mathcal{B}_{r}\right)=(4 \pi)^{(1-m) / 2} \frac{1}{384}\{ & \star \tau+\star \rho_{m m}-7 L_{a a} L_{b b} \\
& \left.+10 L_{a b} L_{a b}\right\}[\partial M] \\
a_{3}\left(\Delta_{1}, \mathcal{B}_{r}\right)=(4 \pi)^{(1-m) / 2} \frac{1}{384}\{ & \star \star \tau+\star \rho_{m m}+(-7 m+116) L_{a a} L_{b b} \\
& \left.+(10 m+104) L_{a b} L_{a b}\right\}[\partial M]
\end{aligned}
$$

The coefficient matrix

$$
\left(\begin{array}{rr}
-7 & 10 \\
-7 m+116 & 10 m-104
\end{array}\right)
$$

has determinant -432 which again is different from zero.

\section{REFERENCES}

[1] T. Branson and P. Gilkey, The asymptotics of the Laplacian on a manifold with boundary, Comm. Partial Differential Equations 15 (1990), 245-272.

[2] P. B. Gilkey, Asymptotic Formulae in Spectral Geometry, CRC Press (2003).

[3] V. K. Patodi, Curvature and the fundamental solution of the heat operator, J. Indian Math. Soc. 34 (1970), 269-285.

[4] J. H. Park, Spectral geometry amd the Kaehler condition for Hermitian manifolds with boundary, Contempory Math (to appear).

[5] R. T. Seeley, Complex powers of an elliptic operator, Proc. Sympos. Pure Math. 10 (1968), 288-307.

[6] —, Analytic extension of the trace associated with elliptic boundary problems, Amer. J. Math. 91 (1969), 963-983.

JHP Dept. of Computer \& Applied Mathematics, Honam University, Seobongdong 59, Gwangsanku, Gwanguu, 506-714 South Korea. Email:Jhpark@honam.ac.kr 